\title{
Emergency Thoracotomy: Indications and Management Challenges in a Developing World
}

\author{
Martins O. Thomas* , Ezekiel O. Ogunleye \\ Lagos University Teaching Hospital, College of Medicine, \\ University of Lagos, Lagos, Nigeria \\ Email: *oluwafemithomas@yahoo.com
}

Received December 10, 2011; revised January 17, 2012; accepted February 15, 2012

\begin{abstract}
Background: Emergency thoracotomies often challenge surgical logistics and they tend to produce inferior outcomes when compared with elective surgery. Aims: We sought to identify the specific indications and therapeutic challenges that go with patients who undergo thoracotomy within 24 hours of admission and the re-thoracotomies. Methodology: Spanning a 7-year period, the bio-data of patients who met our criteria for emergency thoracotomies were collated. We noted their indications for surgery, therapeutic challenges and outcome of care. Results: In all, 36 patients (28 males and 8 females) met the inclusion criteria. Majority, (66.7\%) fell into the 20 - 39 year age range. Diaphragmatic rupture was the commonest indication, followed by massive intra-thoracic haemorrhage. Postoperative mortality occurred in 11.1\% of patients. Postoperative ventilation was absolutely indicated in 6 patients. Discussion and Conclusion: Diaphragmatic rupture is the commonest indication for emergency thoracotomy. We noted the need for improvement in pre hospital care for trauma patients as a way to improve the management outcome of emergency thoracotomies.
\end{abstract}

Keywords: Emergency Thoracotomy; Indications; Definition; Outcome

\section{Introduction}

The subject of emergency thoracotomy has been widely studied worldwide [1-11]. However there has been no dedicated reports on this topical issue from this part of the world. Following Bilroth's 1882 assertion that "the surgeon who should attempt to suture a wound on the heart would lose the respect of his surgical colleagues" [9] (sic), there had been a lot of developments in this all important aspect of care.

The first pre-hospital thoracotomy was performed by Hill in Montgomery, Alabama on a kitchen table in 1902 and since then, with good patient selection, postoperative survival of patients has improved reasonably [9].

Emergency thoracotomy (ET) may serve as a lifesaving tool when performed for the right indications, in selected patients and in the hands of trained surgeons [1]. Critically injured patients "in extremis" arrive at an increasing rate in the trauma bay, as an effect of improved pre-hospital trauma systems and rapid transport [1].

By definition, some authors believe that emergency department thoracotomy encompasses thoracotomies done for patients who are in extremis [2]. Broadly taken, emergency surgery is defined as sternotomy, thoracotomy, laparotomy or major neck vascular repair performed on

*Corresponding author. the day of admission [3]. Operating on a patient might not constitute emergency thoracotomy if the patient was worked up for surgery as an outpatient. The lack of clarity in medical literature, the need for rapid intervention in patients deemed appropriate for the procedure, the life and death nature of the decision and the low but finite functional survival rates following emergency department thoracotomy (EDT) for trauma have made the conduct of these procedures a subject of great controversy among trauma experts [2]. The import of this is that the definition of what constitute emergency thoracotomy is still a subject for further studies till a more universally accepted definition is arrived at. In view of the foregoing, it might be expedient for practicing surgeons to individualise the theory and practice of emergency thoracotomy till that time.

The indications for ET vary widely. In many cases, ET may be indicated for damage control when there is a deadly triad of hypothermia, acidosis and coagulopathy described as temperature of $<34^{\circ} \mathrm{C}$, $\mathrm{pH}$ of 7.2 or less and clinically uncontrollable bleeding [4]. In such situations, the strategy may include the following three steps:

1) Rapid control of haemorrhage and abbreviated surgery in the emergency room or theatre;

2) Correction of hypothermia, acidosis and coagulopathy; and re-evaluation of injuries in the intensive care; 
3) Definitive surgery in the theatre.

Procedures for damage control include aortic crossclamping, hilar clamping, major vessel ligation, pulmonary tractotomy, simultaneously stapled pneumonectomy or lobectomy, cardiac stapling, balloon catheter tamponade, temporary intraluminal shunt, towel packing, towel clip closure, single en masse closure of the chest wall among others.

ET might also be indicated in definitive treatment as in conditions like pericardial tamponade, many cases of intra-thoracic bleeding, systemic air embolism, some lung contusions, lung lacerations and so on. Kaiser and his colleagues [3] at Irvine Medical Centre, California came up with early independent predictions of the need for emergency surgery to include prolonged extrication (odds ratio (OR) 2.3), no loss of consciousness (OR 2.8), intubation (OR 1.7), central line placement (OR1.7) and blood transfusion (OR 2.1) in all $\mathrm{P}<0.05$.

Emergency room thoracotomy saves about one in five patients with isolated penetrating cardiac injury [1]. Multiple studies have shown that patients with isolated penetrating chest trauma have better outcomes than patients who suffer blunt chest trauma without signs of life at the scene or in the emergency department [5]. This is further corroborated by the work of Ahmad and his team in Karachi [6]. In their series, post-thoracotomy mortality was $13.3 \%$ in penetrating injuries and $18.2 \%$ in patients who suffered blunt injuries. In the same series, the combined survival of emergency thoracotomy in 475 patients was $95.58 \%$. They therefore concluded that early recognition of treatable injuries and an aggressive approach in management of patients who need emergency thoracotomy can increase chances of survival of patients suffering from severe chest trauma.

Resuscitative thoracotomy (RT) is a time tested practice that gives direct access to the heart during cardiopulmonary resuscitation. The procedure has been and is still very relevant in modern day practice. RT is also considered to be more successful in penetrating cardiac trauma than in blunt injuries of the heart with cardiovascular collapse [8].

Wise and his colleagues [10] have gone further to simplify instrumentation for ET. Specifically, they stated that a clamshell incision performed using a big scalpel, large scissors, large clamp and a gigli saw will suffice for ET.

In all the works mentioned above, a common phenomenon is the various positions taken by different practitioners on the various aspects of emergency thoracotomy from definition to treatment. Therefore, further literature enrichment would highlight various aspects of ET.

Our study was conducted to highlight the indications and management challenges of ET in a developing coun- try. It was also to further enrich the literature in the all important search for a common ground in the whole subject of emergency thoracotomy.

\section{Methodology}

The study period spanned 7 years from 2000 January to December 2006. A protocol was designed to accommodate details of all thoracotomies performed in the first 24 hours of admission into Lagos University Teaching Hospital, in Nigeria.

We noted patients' bio-data to include ages and sexes, specific indications for surgery, timing, clinical features, operations performed, specific details of postoperative care including complications and interventions.

The cases that met the criteria for emergency thoracotomy were extracted from the lot. Inclusion criteria were thoracotomy performed within 24hours of patient admission and all cases of re-thoracotomies. The patients were followed up on admission taking specific note of postoperative care to include blood transfusion needs, absolute indication for postoperative ventilation and the need for re-thoracotomy. Our criteria for absolute indication for postoperative ventilation was persistently low $\mathrm{SpO}_{2}(<80 \%)$ with intra-nasal oxygen.

The data so collected were analysed normally looking at population distribution, dispersion, correlation of postoperative events (mortality) with elective or emergency thoracotomy.

\section{Results}

There were 36 patients for emergency thoracotomies within the study period. They comprised of 28 males and 8 females giving a male to female ratio of 3.5:1. Within the 20 - 39 year age range, there were 24 patients constituting $66.7 \%$ of the population. Age 19 and below produced only 5 patients constituting $13.9 \%$ of the cases.

The mean age was $33.7 \pm 12.1$ years and the median was 32.1years. The mean and median were fairly close in value giving a tendency to being a normal distribution but the distribution was fairly platykurtic with Pearson Skewness of +0.39 .

Massive Haemothorax was the indication in 11 (30.5\%) patients while diaphragmatic rupture occurred in 15 (41.7\%) patents. Re-thoracotomy was the reason in 3 patients. Thoracotomy was done for 35 patients while sternotomy was done for one patient who suffered penetrating cardiac trauma. Postoperative mortality occurred in $4(11.1 \%)$ of the patients. There were absolute indications for postoperative ventilation in 6 (16.7\%) patients.

\section{Discussion}

In emergency situations, thoracotomy is done in most 
cases as a major therapeutic intervention. It is resorted to for quick reversal of worsening situations like major chest trauma, cardiac arrest and other similar conditions.

What constitute ET has been described in literature by various authors [1-8]. There seems to be convergence of opinion that ET as a procedure is performed within 24 hours of admission. This definition however is not appropriate for re-thoracotomy which is also a type of ET that may follow a well planned elective surgery done days after admission. A patient whose elective surgery workup was done as outpatient can be operated upon within 24 hours of admission. There is also the issue of ET in damage control [4]. In this situation, ET is done for patients in clinical extremis with survival as the main consideration.

Resuscitative thoracotomy (RT) is another form of ET which is indicated for open cardiac massage in cardiopulmonary resuscitation.

With this background information, the indications for ET can be summed up to include all the indications for the component parts of ET (vide supra).

The preponderances of indications for emergency thoracotomy (ET) seem to be different in various localities $[1,2,6,7]$ In our study with 36 patients, diaphragmatic rupture was the commonest indication for ET while massive haemothorax was next (Table 1). These two indications constituted $72.2 \%$ of the cases. Even at that, the main reason for doing thoracotomy for diaphragmatic rupture was when additional intra-thoracic injury was suspected or when diaphragmatic rupture was an incidental finding during thoracotomy. Aside these, we maintained the practice of repairing the diaphragm through the abdomen in acute cases.

Ahmad and his colleagues [6] working in Karachi noted that massive haemothorax was the commonest indication in their series. Even at that, haemothorax only occurred in $10.9 \%$ of the series and this is about a third of the massive haemothorax rate in our study.

Another indication of interest is re-thoracotomy. This was done in $8.3 \%$ of cases. One was done for postoperative cardiac arrest while the remaining were done for continuing haemorrhage after thoracotomy. It is important to note that there was no case that rightly met the

Table 1. Indications for emergency thoracotomy.

\begin{tabular}{lccc}
\hline \multicolumn{1}{c}{ Indications } & Males & Females & Total \\
\hline Massive Haemothorax & 8 & 3 & $11(30.5 \%)$ \\
Penetrating cardiac injuries & 1 & - & $1(2.8 \%)$ \\
Diaphragmatic rupture & 12 & 3 & $15(41.7 \%)$ \\
Re-thoracotomy & 2 & 1 & $3(8.3 \%)$ \\
Others & 5 & 1 & $6(16.7 \%)$ \\
\multicolumn{1}{c}{ TOTAL } & $28(77.8 \%)$ & $8(22.2 \%)$ & $100(100.0 \%)$ \\
\hline
\end{tabular}

Table 2. Age and sex distribution.

\begin{tabular}{cccc}
\hline Age (Years) & Males & Females & Total \\
\hline$<10$ & 1 & 2 & $3(8.3 \%)$ \\
$10-19$ & 2 & - & $2(5.6 \%)$ \\
$20-29$ & 9 & 2 & $11(30.6 \%)$ \\
$30-39$ & 10 & 3 & $13(36.1 \%)$ \\
$40-49$ & 4 & 1 & $5(13.8 \%)$ \\
$50-59$ & 2 & - & $2(5.6 \%)$ \\
Total & $28(77.8 \%)$ & $8(22.2 \%)$ & $36(100.0 \%)$ \\
\hline
\end{tabular}

criteria for damage control as described by Mashiko and his team [4] This might be a reflection of poor pre-hospital care in this environment. Patients who qualified for this might have died at the scene or they could have been brought in dead.

From our study, $77.8 \%$ of patients were males while the remaining 22.2\% were females (Table 2). The preponderance of males over the females eased a bit in the groups below 20 years of age. A plausible explanation is the general trend of males being more mobile and adventurous that they get involved in trauma than their female counterparts. In all, age range 20 - 39 years constituted $66.7 \%$ of all cases. This again is the usually very active age group that is relatively more exposed to trauma.

There were absolute indications for postoperative ventilation in $16.7 \%$ of our patients. Post-thoracotomy mortality occurred in $11.1 \%$ of our series. This is a bit lower than $15.3 \%$ in the Karachi report [6]. This is despite the poor pre-hospital care in our environment [12]. The implication of this is that the patients with major chest trauma and many others who could benefit from ET would have died at the pre-hospital level.

We concluded that diaphragmatic rupture is the commonest indication for emergency thoracotomy because of the frequent suspicion of additional intra-thoracic injuries. This is followed closely by massive intra-thoracic haemorrhage.

We recommend further improvement in pre-hospital care of trauma patients so that more patients can be saved through emergency thoracotomy.

\section{REFERENCES}

[1] K. Soreide, P. Petrone and J. A. Asencio, "Emergency Thoracotomy in Trauma: Rationale, Risks and Realities," Scandinavian Journal of Surgery, Vol. 96, No. 1, 2007, pp. 4-10.

[2] J. C. Mejia, R. M. Stewart and S. M. Cohn, "Emergency Department Thoracotomy," Seminars in Thoracic and Cardiovascular Surgery, Vol. 20, No. 1, 2008, pp. 13-18. doi:10.1053/j.semtcvs.2008.01.005

[3] M. Kaiser, P. Ahearn, X. M. Nguyen, A. Barleben, M. 
Cinat, et al., "Early Predictors of the Need for Emergency Surgery to Control Haemorrhage in Hypotensive Trauma Patients,” American Surgery, Vol. 75, No. 10, 2009, pp. 986-990.

[4] K. Mashiko, H. Matsumoto, T. Mochizuki, K. Takuhiro, Y. Hara, et al., "Damage Control for Thoracic Injuries," Nippon Geka Gakkai Zasshi, Vol. 103, No. 7, 2002, pp. 511-516.

[5] J. Menaker, J. Cushman, J. M. Vermillion, R. E. Rosenthal and T. M. Scalea, "Ultrasound-Diagnosed Cardiac Tamponade after Blunt Abdominal Trauma Treated with Emergency Thoracotomy," Journal of Emergency Medicine, Vol. 32, No. 1, 2007, pp. 99-103. doi:10.1016/j.jemermed.2006.05.036

[6] T. Ahmad, S. W. Ahmed, N. Hussain and M. I. Khan, "Role of Urgent Thoracotomy in Improving the Survival of Patients with Severe Chest Trauma," Journal of College Physicians and Surgeons Pakistan, Vol. 19, No. 9, 2009, pp. 575-578.

[7] M. Ben khadra, D. Honnart, F. Lenfant, P. Trouilloud, C. Girrard and M. Freysz, "Open Cardiopulmonary Resuscitation: Is There an Interest in France?” Annales Fran- çaises d'Anesthésie et de Réanimation, Vol. 27, No. 11, 2008, pp. 920-933.

[8] M. Kalima, E. Teeple and G. Fulda, "Are There Still Selected Applications for Resuscitative Thoracotomy in the Emergency Department after Blunt Trauma?” Delaware Medical Journal, Vol. 81, No. 5, 2009, pp. 195-198.

[9] B. Karim, "Emergency Department Thoracotomy: Indications and Techniques of Resuscitative Thoracotomy," An Online Publication, September 2006. www.trauma.org

[10] D. Wise, G. Davies, T. Coats, D. Lockev, J. Hyde and A. Good, "Emergency Thoracotomy: 'How to Do It'," Journal of Emergency Medicine, Vol. 22, No. 10, 2005, pp. 22-24.

[11] C. C. Cothren and E. E. Moore, "Emergency Department Thoracotomy for the Critically Injured Patient: Objectives, Indications and Outcomes," World Journal of Emergency Surgery, Vol. 1, No. 4, 2006, pp. 1-4.

[12] M. O. Thomas and E. O. Ogunleye, "Penetrating Chest Trauma in Lagos Nigeria,” Asian Cardiovascular \& Thoracic Annals, Vol. 13, No. 2, 2005, pp. 103-106. 